\title{
AN EFFICIENT WAY OF FINDING POLARITY OF ROMAN URDU REVIEWS BY USING BOOLEAN RULES
}

\author{
HALIMA SADIA, MOHIB ULLAH, TARIQ HUSSAIN, NIDA GUL, MUHAMMAD FAROOQ HUSSAIN, NAUMAN UL \\ HAQ, AND ABU BAKAR
}

\begin{abstract}
Opinion mining is the technique of analyzing the sentiment, behavior, feelings, emotions, and attitudes of customers about a product, topic, comments on social media, etc. Online shopping has revolutionized the way customers do shopping. The customer likes to visit the online store to find their product of interest. It is becoming more difficult for customers to make purchasing decisions solely based on photos and product descriptions. Customer reviews provides a rich source of information to compare products and make purchasing decisions commonly on the basis of other customer reviews. Clients provide comments in the language of their choice, e.g. the people of Pakistan use Roman script based the Urdu language. Normally such comments are free from scripting rules. Hundreds of comments are given on a single product, which may contain noisy comments. Identifying noisy comments and finding the polarity of these comments is an active area of research. Limited research is being carried out on roman Urdu sentiment analysis. In this research paper, we propose a novel approach by using Boolean rules for the identification of the related and non-related comments. Related reviews are those which show the behavior of a customer about a particular product. Lexicons are built for the identification of noise, positive and negative reviews. The precision of the evaluation results is $68 \%$, recall is also $68 \%$ and F-measure is 68 The accuracy of the whole evaluation is $60 \%$.
\end{abstract}

Key words: Roman Urdu, Polarity, Boolean rules, Opinion Mining, Noise, Reviews polynomial

AMS subject classifications. 90C09

1. Introduction. Sentiment analysis methodologies are proposed for the classification of opinions in multiple languages. Opinion mining also known as sentiment mining is a process of identifying opinions from any language. Opinion mining is a combination of data mining and natural language processing techniques [1]. In [2] define opinion mining as the process that searches results of a given product and produce a list of their reviews attribute. Customer opinion mining is the process of checking customer interest in a particular product [3]. Opinion mining is a technique of analyzing reviews of customers which helps both the customer and retailer. The retailer easily identifies customer interest and demand for products from these reviews. Another angle of opinion is customer convincing customers [4]. Most of the customers can make their purchasing decision based on customer opinions present on the retailer website [5]. It is time-consuming to extract important information from multiple reviews over one product. The Internet is an important part of our daily life. Internet brings us a lot of advantages; online shopping is one of them, it easier for the customers to buy goods or services from a vendor. Consumer finds their product of interest by visiting the vendor's website[6]. It is difficult for customers to use Urdu based keyboards as most handheld devices need software updates and installations packages for using Urdu languages [7]. The customers mostly rely on the Roman script for Urdu which is easily available but unfortunately with no scripting structure. Normally Urdu language written in Roman script is called Roman Urdu. Let us take an example of Roman Urdu phrase" ye mobile bht mehnga he" or "ye mobile mene istemal kia he bht acha h". On shopping sites over internet, customers use their native languages for posting their reviews and these reviews are understood by few people, mining useful information from these reviews become a difficult task [8][4]. Especially, it creates a problem for non-native users to understand these reviews and to obtain useful information. Another main issue is syntax and the changing meaning of words in different situations. Some words show positive meaning in one situation and negative in another[9]. For example, "ye mobile acha he" in this sentence the word "acha" shows that mobile is good. If we say ye mobile "acha nahi" he

\footnotetext{
*Institute of Computer Science and Information Technology, The University of Agriculture Peshawar 25130, Pakistan. (uom.tariq@gmail.com)
} 
in this sentence the word "acha" is the same but the word "nahi" changing the semantics change and that means mobile is not good. Another important issue is the use of several syntax for the same word by the different users e.g. multiple users will use roman urdu word" acha" meaning "good" in Engllish with different syntax like "icha" or "echa" etc. So, it is difficult to handle these reviews according to its orientation [10] [11]. Also, if the sentence contains both positive and negative opinion words like" ye mobile acha nahi he" here the word "acha" is positive, and "nahi" is negative so the opinion is negative, but they consider it as positive only because of the word "acha". Customer reviews are posted in different native languages. NLP techniques which enable computers to understand human natural languages. Linguistics tasks are performed through NLP such as translation, summarization, analyses, Part-of-Speech (POS) tagging, information retrieval, speech recognition [12][13]. In opinion mining, POS tagging techniques plays a vital role as it is used to identify different features word and opinion sentence in opinion repository. Many types of lexicons are available for word matching and feature identifications. Many researchers have worked on opinion mining and proposed different models. But most of them worked over English, Chinese, Arabic, Urdu, Pashto and Sindhi, etc [14][17]. Urdu is the national language of Pakistan and is like the Hindi language. Both the languages have different scripts but having some pronunciations. They are mostly used in South East Asia with nearly 1.5 billion populations [10][18]. Users also use Roman-Urdu on social media when communicating with their friends and posting their comments on different blogs and websites. This proposed model is used to identify the polarity of customer reviews written in Roman Urdu by applying proposed Boolean rules in form of precision, recall, F-measure and accuracy.

This research paper has several contributions:

- With the emerging power of internet online shopping has become more attractive to customers. Customer likes to buy products online, but before buying the products they visit reviews given by other customers. Reviews play an important role in buying decisions of a customer. Customer post their reviews in the language of their choice, in Pakistan people normally post their reviews in Roman Urdu. Roman Urdu is unstructured free text. It is observed from the customer reviews that there are many noisy comments in the review list Identifying customer reviews as noise or related comments and finding the polarity of Roman Urdu comments is a research issue. Another problem is that two negative words change the polarity of a review, this is an ultimate need to identify and categorize those comments. In this research is going to identify noisy and related comments and find the polarity of those comments.

- The paper provides how to identify noise in user reviews? "In this research discuses how to find polarity of roman Urdu reviews?

- In this research proposed to identify related and noisy comments from Roman Urdu reviews "

- Introduces state of the art model to find the polarity of comments using proposed Boolean Rules.

In the next section 2, the literature review of the Roman Urdu and boolean rules. In section 3, boolean rules Principles and Categories is explained. Section 4 explains the results evaluation. Section 5 provides the experimental results of the roman urdu language by using boolean rules. And finally, conclusions, recommendation and future work is explained in section 6 and 7.

2. Related works. The authors in [19] have worked on opinion mining. They tried to mine all opinions of customers and summarize them. Their summarization task was different from traditional summarization because they only mine the features of a product on which the customer has expressed their opinions. First, they mine the product features which are commented by the users, secondly identify the opinion sentence and its polarity and third summarizing the results. The experimental results show that techniques are more effective. The research conducted in [12] has presented their work in the Sindhi language. They proposed a technique for part of speech tagging. And develop a linguistic rule for the Sindhi language. The orthography of Sindhi language is difficult due to the absence of diacritic symbols. For the development of the Sindhi Part of Speech (SPOS) tagging system, they used a supervised approach. They set 186 disambiguation rules for POS tagging. The POS tagging algorithm take words and check into the lexicon if the word were present in the lexicon, the associated tag was assigned and if the word was not presented in the lexicon then tag set was assigned according to linguistic rules. The output of the system was Sindhi word, in English, and the Sindhi language. In [20] present models for finding the polarity of tweets. They build a two-way task for classifying the polarity of tweets as positive and negative and three-way tasks for finding polarity as positive, negative, 
and neutral. They utilize three types of models for their experiment i.e. unigram,feature-based and tree kernel model. As a baseline, they used the n-gram model, for the feature-based model they used for tree kernel base model they design a tree of tweets. Their experimental results for a two-way task shows that unigram model is a hard baseline which only achieve $20 \%$ in both tasks and the feature-based model achieve similar accuracy as the unigram model, but the tree kernel model achieves the accuracy of $2.58 \%$ and $2.66 \%$ over these two models. They also experimented with these models in combination. Combining unigrams with feature models achieved $0.78 \%$ over the combined model of the feature model with tree kernel. For all their experiment they used SVM (support vector machine). Experimental results for a three-way task shows that tree kernel model achieved more accuracy form unigrams and baseline model. It achieved $4.02 \%$ accuracy over unigram model and $4.29 \%$ over Senti-feature model. In, [21] proposed a system for mining opinion written in Arabic language. The proposed method was the combination of three methods i.e. lexicon-based method, maximum entropy, and KNN method. Set of documents were classified by using a lexicon-based method for training machine. Then for the maximum entropy method, these classified documents act as a training set. Further other documents are classified as maximum entropy. For classification of the rest documents that output of the two methods was used as a training set for the k-nearest method. Experimental results showed that $50 \%$ when only the lexicon-based method was applied to the dataset it gives 50\% accuracy which exceeded to $60 \%$ when lexicon and entropy-based methods were applied in the combination and $80 \%$ when three methods were applied together. In,[22] worked on Punjabi text classification. They proposed a hybrid system by blending Naïve Bayesian and N-grams. They extract the features of $\mathrm{N}$-grams and used it to train Naïve Bayesian. They then validated the trained model using testing data. Experimental results were compared with existing models and results from comparison show a better efficiency of the proposed method. In [23] proposed for text classification techniques for Roman-Urdu reviews by using the Waikato Environment for Knowledge Analysis (WEKA). With the emerging use of the internet and e-commerce, opinion mining and sentiment analysis become a very important field for both researchers and retailers. They create a data set of 150 positive and 150 negative reviews for training machine. Naïve Bayesian, Decision Tree and KNN classification models were designed to analyze the polarity of new customer reviews based on trained data set. Their result shows that and F-measure and Naïve Bayesian performed better than Decision Tree and KNN classification models in terms of accuracy, precision, recall. In, [4] proposed a method in which they provide a facility to the non-Urdu speaking customers to get benefit from the comment posted in Roman Urdu. They took the data from a website called WHATMOBILE where people give there-reviews over a phone they want to buy or been using. They define their work in to 4 main steps. They 1st made a Crawler which take the comments from the site which contain both useful and noisy comments, Then they use BING Translator to translate the comments from roman Urdu to English to make the computer understand, they then extract their opinion the relevant comments by using POS and preprocessing and removing the noise and use their local database for the Identification of the opinion polarity and at last show the user reviews and rating in graphical form. The experimental results recorded $27 \%$ precision and categorized $21.1 \%$ reviews falsely due to noisy data.

3. Proposed method. This model consists of five steps. First, reviews written in roman Urdu are extracted from the mobile website whatmobile.com written in Roman-Urdu and are collected in a word document. These reviews are positive, negative, and noisy. Different lexicons for positive, negative, and for noise identifying are created for testing and results. After the data processing, the stopping words are removed from the reviews. The reviews are then parsed according to the noise dictionary and noise is removed. After removing noise, the reviews are parsed for identifying the polarity of reviews through Boolean Rules.

Rule no 1 According to AND gate, when there is one positive and one negative word exist in a review then the polarity of the review will be negative.

Rule no 2 According to the XNOR gate, when two negatives exist in a review then the polarity of the review will be positive.

Rule no 3 According to the XNOR gate, when two positive words are existing in a review then the polarity of the review will be positive.

Rule no 4 According to AND gate, when one negative and one positive word exists in a review then the polarity of the review will be negative.

Rule no 5 If there is a single positive in a review then the review will be positive. 
Rule no 6 If there is a single negative exists in a review then the review will be negative.

3.1. Preprocessing. In this step, noisy data present in the reviews is eliminated. Irrelevant views like comments which do not have any positive or negative opinion about products will be categorized as noisy data. Lexicon of noise is created to identify noise from reviews.

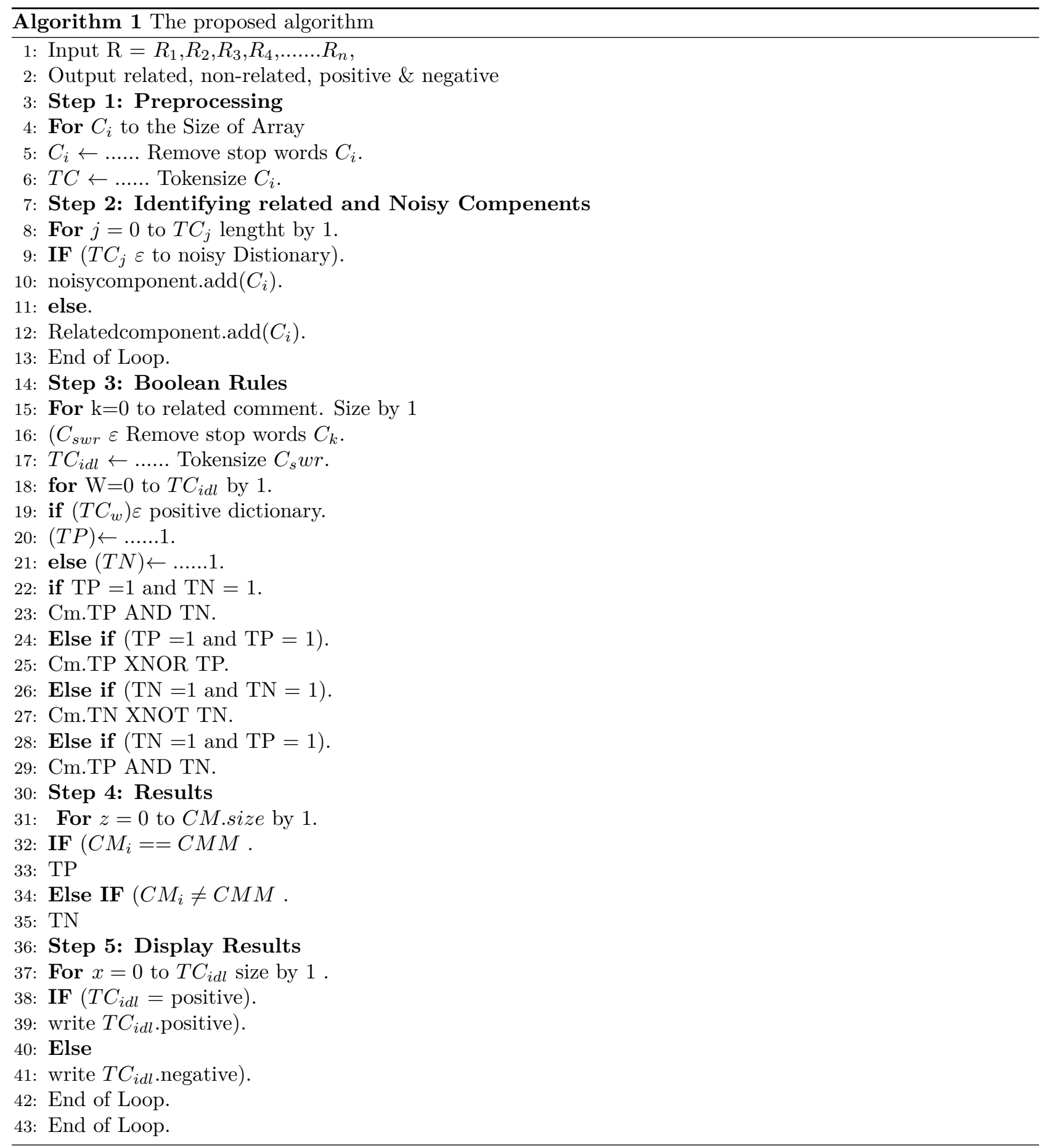




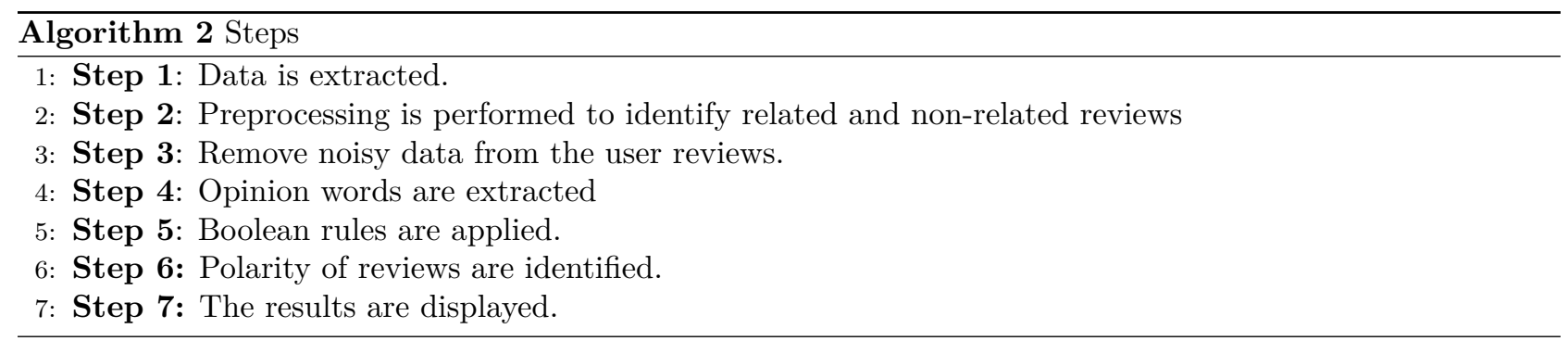

TABLE 3.1

Original Data Set 1st January 2016 to 31st December 2017

\begin{tabular}{|c|c|c|c|}
\hline Total Reviews & $\mathbf{4 5 4}$ & Positive & Negative \\
\hline Samsung mobile & 82 & 51 & 29 \\
\hline Nokia mobile & 96 & 61 & 36 \\
\hline Q mobile & 170 & 75 & 93 \\
\hline Noise & 106 & & \\
\hline
\end{tabular}

Data set. Reviews in roman Urdu are crawled from website whatmobile.com in the time span of 1st January 2016 to 31st December 2017. Three types of mobiles are selected. Samsung, Nokia, and Qmobile. A total of 454 reviews are crawled. These reviews are consisting of positive, negative, and noisy. Positive reviews are those which show some positive opinion about a product e.g. "ye mobile bht acha h". Negative reviews are those which shows negative opinion about a product e.g. "bht bakwaas mobile he ye". Noisy reviews are those reviews which are not related e.g. "me ye mobile bechna chahta hun agr koi lena chahy to". All the product reviews are manually evaluated, and the result is calculated. This research aims to find the polarity of reviews about products and not to criticize or demotivate any product.

3.2. Noise Lexicon. Table 3.2 shows noise lexicon and noisy comments. The lexicon consists of terms that are used to identify or extract the noise from user opinions. The lexicon is generated by identifying the words from the noisy reviews. As roman Urdu has no rules for writing reviews so the users have used different writing styles and spellings for the same word to express their opinion e.g. warranty, woranti, warenti etc.

In this step, the opinion words are extracted from the refined reviews. The opinions are identified through these words. The opinion words are stored in the opinion word lexicon.

Positive lexicon. Table 3.3 consists of positive lexicon and positive words. The lexicon consists of words that are used to identify positive reviews. Similarly like in noisy reviews users also have used different writing styles in positive reviews e.g. zabardast, zberdast, zbardast etc.

Negative lexicon. Table 3.4 consists of negative lexicon and negative reviews. A negative lexicon consists of words that are used to identify negative reviews. Like positive lexicon, negative lexicon has words with different writing styles and spelling e.g. bekaar, bekar, bkwaas, bakis etc.

3.3. Applying Boolean Rules. In this step, the Boolean rules are applied to the reviews to identify the polarity. Figure 3.1 shows Boolean rules of all required gates.

3.4. Identification. After applying the Boolean rules, the opinion sentence is identified.

4. Result evaluation. The results of the proposed model are evaluated by using standard methodologies of information retrieval i.e. precision, recall, F-measure, and accuracy. Following 2 by 2 confusion matrix which is also called contingency, the matrix is used to evaluate measurements. A confusion matrix [24] includes information on current and anticipated classifications made through a rating model. The operation of such a model is usually measured using the matrix data. The confusion matrix is also called a contingency table. The entries within the confusion matrix cover the following meaning in the background of our study shown in table 4.1 .

5. Experimental Results. This section contains the experimental results of the model. For experimental reasons, users' opinions are collected on the "whatmobile.com" website. More than 400 reviews of NOKIA, 
TABLE 3.2

Noise Lexicon

\begin{tabular}{|c|c|}
\hline Noise lexicon words & Reviews \\
\hline \multirow{3}{*}{ Warranty } & mery pas lumia 530 hy warranty \\
& nhi hy full box hy kisi kochahye \\
& to msg kry no call only sms plzz final \\
& 4200 iam frommultan 03012939265 \\
\hline \multirow{2}{*}{ Sale } & Me j5 mobile sale krna chata ho. Condition9/10 \\
& white colorprice in 16000 location \\
& Faisalabad sell no03117215820 \\
\hline
\end{tabular}

TABLE 3.3

Positive lexicon

\begin{tabular}{|c|l|}
\hline Positive lexicon words & \multicolumn{1}{c|}{ Positive Reviews } \\
\hline Zabardast & $\begin{array}{l}\text { Zabardast mobile habattery timing ny } \\
\text { to tamam smart phones kopechy chor dia }\end{array}$ \\
\hline Sale & $\begin{array}{l}\text { Outclass mobile he. aik dinbatrery backup. } \\
\text { camera front and backbht acha h }\end{array}$ \\
\hline
\end{tabular}

SAMSUNG, and Qmobile have been posted from the website in spam from January 1, 2016, to December 31, 2017, to identify polarity. The data has been collected into an MSWord document. Dictionaries for positive, negative, and noise identification have been created for testing and results. For evaluation purposes, each review is manually analyzed and checked if it is positive, negative, or noise. Manually observed results are then compared with practical results. A total of 104 non-related or noisy reviews were crawled and 103 are identified successfully. The percentage of noisy reviews was $99.30 \%$. Lexicon of noise is created to extract noisy comments from the reviews. There were a total of 454 reviews of three products, total positive reviews were 186 and practically 163 were evaluated positive, total negative were 164 and practically 153 were identified as negative, and total 104 were non-related comments and 138 were identified correctly. The results show that $35 \%$ of reviews are identified positively, $33 \%$ as negative, and $30 \%$ noise of total reviews. Table 5.1 shows the noisy comments observed during reviews crawling.

While the TP is true positive comments i.e. number of all actual positive. TN is true negative is the total number of negative comments. FP is the total number of negative comments that are retrieved as positive. While FN is a total number of comments which were positive but practically, they are identified as negative. Precision recall and F-measure and accuracy have been calculated by using the contingency Table 5.4.

Boolean rules. Table 5.5 shows Boolean rules were created to identify the polarity of reviews on the basis of positive and negative words that exist in the reviews.

Lexicons. Three types of lexicons will be created to identify noise and to find the polarity of reviews. Table 5.6 shows no of words of all lexicons. All of the lexicons are consist of following no of words.

5.0.1. Noise Lexicon. Table 5.7 shows noise lexicon and noisy comments.

Three types of lexicons are created to identify noise and to find the polarity of reviews.

Positive lexicon. Table 5.8 consists of positive lexicon and positive words.

Negative lexicon. Table 5.9 consists of negative lexicon and negative reviews.

5.1. Individual Products Result. In the case of the first product, out of 82 , there were 18 true positives, 19 true negatives, 14 false positives, and 31 false negatives. Table 5.10 shows the contingency matrix of Samsung mobile. Figure 5.2 shows the graph of Samsung mobile. Table 5.10 contingency matrix of Samsung mobile

In the case of second product total, 96 comments were crawled, out of 96 there were 33 true positives, 23 true negatives, false-negative 27 , and 15 were false positive. Table 5.12 shows the contingency matrix of the Nokia mobile. Figure 5.2. shows the contingency matrix graph of the Nokia mobile.

In the case of the third product total, 170 reviews were crawled. Out of total 170, the true positive rate was 45 , the true negative was 29 , false-negative 32 and false-positive were 64 . Table 5.14 shows the contingency matrix of Qmobile. Figure 5.3 shows the contingency matrix graph of Qmobile. 
TABLE 3.4

Negative lexicon

\begin{tabular}{|c|l|}
\hline Negative lexicon words & \multicolumn{1}{|c|}{ Negative Reviews } \\
\hline bura & $\begin{array}{l}\text { yar ya mobile kisi kamka nai hay bht hi } \\
\text { bura hay }\end{array}$ \\
\hline Bekar & $\begin{array}{l}\text { full time bekar set ha yah 3.30ghantylaita ha } \\
\text { charging ma ... Paisy brbad krny ha tw lylo }\end{array}$ \\
\hline
\end{tabular}

\begin{tabular}{|c|c|c|c|c|}
\hline \multirow{2}{*}{ Rule no 1 } & \multicolumn{2}{|c|}{ Inputs } & Output (AND) & Equation \\
\cline { 2 - 5 } & $\mathrm{A}$ & $\mathrm{B}$ & $\mathrm{C}$ & \\
\cline { 2 - 4 } & Positive & Negative & Negative & \\
\hline
\end{tabular}

(a) Boolean Rule 1 for (AND Gate) Equation

\begin{tabular}{|c|c|c|c|c|}
\hline \multirow{2}{*}{ Rule no 2 } & \multicolumn{2}{|c|}{ Inputs } & Output (XNOR) & \multicolumn{1}{c|}{ Equation } \\
\cline { 2 - 5 } & $\mathrm{A}$ & $\mathrm{B}$ & $\mathrm{C}$ & $\mathrm{C}$ \\
\hline
\end{tabular}

(b) Boolean Rule 2 for (XNOR Gate) Equation

\begin{tabular}{|c|c|c|c|c|c|}
\hline \multirow{3}{*}{ Rule no 3} & \multicolumn{2}{|c|}{ Inputs } & Output (XNOR) & Equation & \\
\hline & A & B & $\mathrm{C}$ & & \\
\hline & Negative & Negative & Positive & & C \\
\hline
\end{tabular}

(c) Boolean Rule 3 for (XNOR gate) Equation

\begin{tabular}{|c|c|c|c|c|}
\hline \multirow{2}{*}{ Rule no 4} & \multicolumn{2}{|c|}{ Inputs } & Output (AND) & Equation \\
\cline { 2 - 5 } & $\mathrm{A}$ & $\mathrm{B}$ & $\mathrm{C}$ & \multirow{2}{*}{$\mathrm{A}$} \\
\cline { 2 - 5 } & Negative & Positive & Negative & $\mathrm{C}$ \\
\hline
\end{tabular}

(d) Boolean Rule 4 for (AND gate) Equation

\begin{tabular}{|c|c|c|c|}
\hline \multirow{3}{*}{ Rule no 5 } & Inputs & Output & Equation \\
\cline { 2 - 3 } & $\mathrm{A}$ & $\mathrm{C}$ & \\
\cline { 2 - 3 } & Positive & Positive & $\mathrm{A}$ \\
& & & \\
\hline
\end{tabular}

(e) Boolean Rule 5 for single positive Equation

\begin{tabular}{|c|c|c|c|}
\hline \multirow{3}{*}{ Rule no 6 } & Inputs & Output & \multicolumn{1}{|c|}{ Equation } \\
\cline { 2 - 3 } & $\mathrm{A}$ & $\mathrm{C}$ & \\
\cline { 2 - 4 } & Negative & Negative & $\mathrm{A}$ \\
\hline
\end{tabular}

(f) Boolean Rule 1 for single Negative Equation

FIG. 3.1. Rules for Logic Gates

TABLE 4.1

Result evaluation

\begin{tabular}{|c|c|c|c|}
\hline & \multicolumn{2}{|c|}{ Prediction } \\
\hline & & Negative & Positive \\
\hline \multirow{2}{*}{ Actual } & Negative & $\mathrm{TN}$ & $\mathrm{FP}$ \\
\hline & Positive & $\mathrm{FN}$ & \\
\hline
\end{tabular}




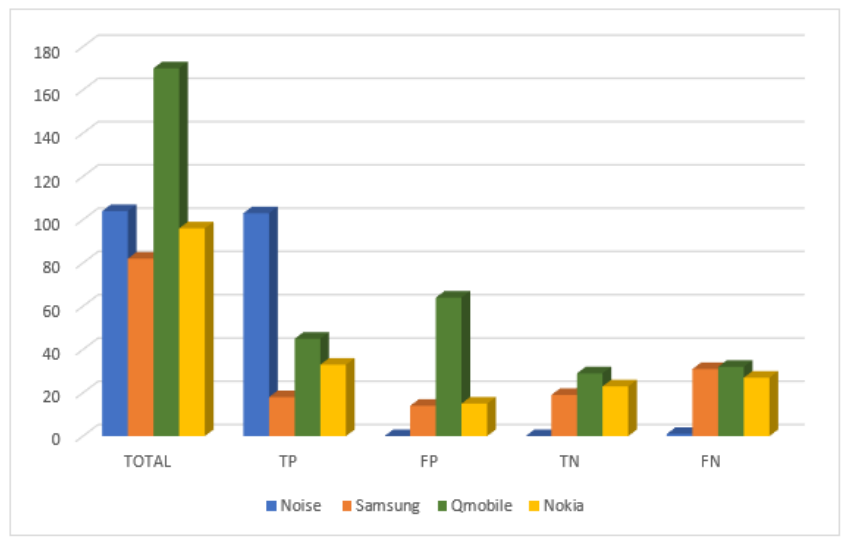

FIG. 5.1. Contingency matrix graph of All Reviews

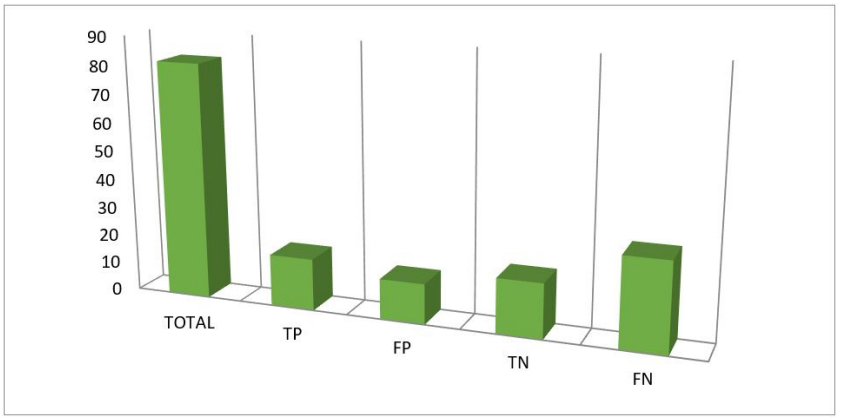

FIG. 5.2. Contingency matrix graph of Samsung mobile

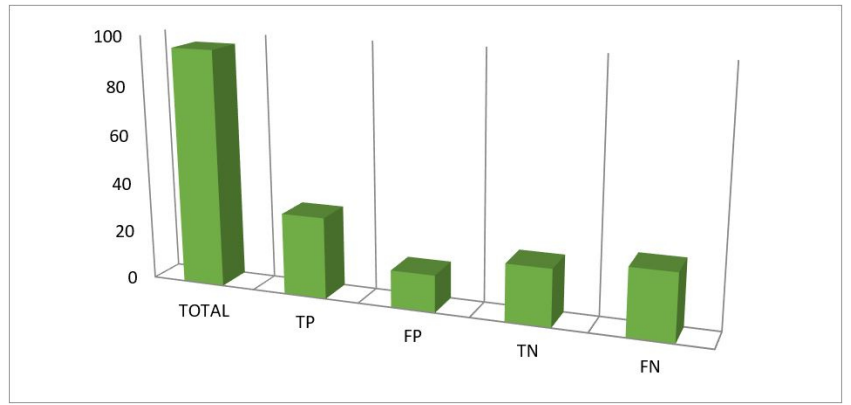

FIG. 5.3. Contingency Matrix Graph of Nokia Mobile

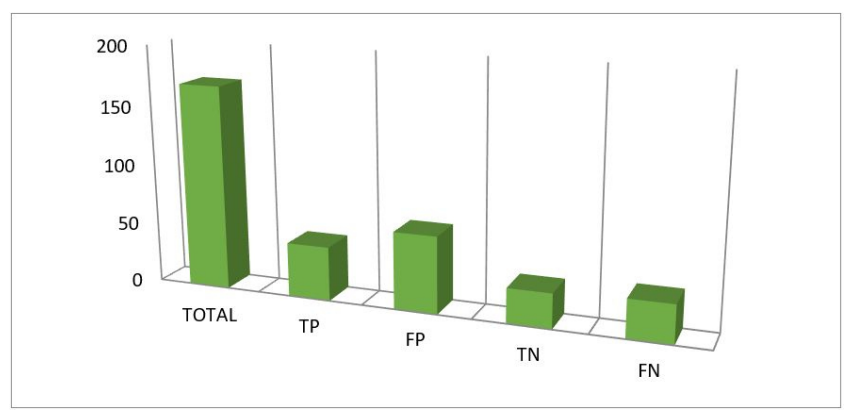

FIG. 5.4. Contingency matrix graph of Qmobile 
TABLE 5.1

Related and non-related comments

\begin{tabular}{|l|l|}
\hline \multicolumn{1}{|c|}{ Related comments } & \multicolumn{1}{c|}{ Nonrelated Comments } \\
\hline $\begin{array}{l}\text { Bakwass tareen cell phone hai } \\
\text { guys, ye acha nahih, Aj mujhy }\end{array}$ & \\
$\begin{array}{l}\text { S8 purchase key howy 2 din } \\
\text { howyJaib sy giraor glass breake } \\
\text { ho gaya yani Gorillaglass ki } \\
\text { wat lag gai or waranty green } \\
\text { touch waly1 mah bad clame ker } \\
\text { k daingy or 10\% amount bhily gy }\end{array}$ & $\begin{array}{l}\text { koi falt nhihai condition } 9.5 / 10 \\
\text { haii only } 18000 \text { SeriesPerson contact } \\
\text { me at this } \backslash \backslash \text { nmber. 03558153892 }\end{array}$ \\
$\begin{array}{l}\text { Yar ya mobile kisi kam ka nai hay } \\
\text { bht hi bura hay }\end{array}$ & $\begin{array}{l}\text { Agar kisi k pass J5 (15) dead halatmain } \\
\text { hu jiskiLCD thik hu grwo sale krna chahy } \\
\text { tu plzRabtakrain... 0300-4746675 }\end{array}$ \\
\hline
\end{tabular}

TABLE 5.2

Total results of all products

\begin{tabular}{|l|l|l|l|l|l|l|}
\hline \multirow{2}{*}{} & \multicolumn{2}{|c|}{ Noise } & \multicolumn{2}{c|}{ Positive } & \multicolumn{2}{c|}{ Negative } \\
\cline { 2 - 7 } & Reviews & Percentage & Reviews & Percentage & Reviews & Percentage \\
\hline $\begin{array}{l}\text { Practical } \\
\text { Evaluation }\end{array}$ & 138 & $30.0 \%$ & 163 & $35.0 \%$ & 153 & $33.0 \%$ \\
\hline Manually & 140 & $22.90 \%$ & 186 & $40.97 \%$ & 164 & $36.12 \%$ \\
\hline
\end{tabular}

5.2. Combine Products Result. TP is true positive comments i.e. number of all actual positive. TN is true negative is the total number of negative comments. FP is the total number of negative comments that are retrieved as positive. While FN is a total number of comments which were actually positive but practically, they are identified as negative. Table 5.16 shows the contingency table results of all products.

6. Discussion. The experimental results show that the model identified the related and non-related comments successfully. Lexicons of noise, positive and negative are generated for identification of noise and finding polarity of reviews. Boolean rules are generated to identify the polarity of reviews. The work has been evaluated in two steps. In the first step, the noisy comments are identified with the help of noise lexicon while in the second step the Boolean rules are applied on reviews, and by the lexicon of positive and negative the reviews are identified successfully. The true positive rate was 199, false-positive was 93 , the true negative was 70 and false negative was 91 . The precision of the whole evaluation is 0.68 , recall is $0.68, \mathrm{~F}$-measure is 0.67 , and accuracy is 0.60 . All products are also evaluated individually and this time the true positive 96 , false-positive 93 , true negative 70 , and false negative is 90 . The precision is 0.507 , recall is 0.516 , F-measure is 0.511 , and accuracy is 0.407 .

7. Conclusion. Opinion mining is a vast and challenging area of research as it deals with natural language. Due to the complexity of human language, it is very difficult to obtain useful information. Human language has no such rules of typing that's why users can type the same word with a different style. Roman Urdu is also a simple way of conveying a message. The majority of people in Pakistan convey their message in roman Urdu, but not enough work has been done on roman Urdu. The paper proposed state of the art opinion mining parser is generated by using Boolean rules model to find the polarity of customer reviews that are posted on the different retailer websites. Different lexicon of for identification of noise, positive and negative reviews. In this research the experimental results show that practical evaluation was mainly based on lexicons. The precision of practical evaluation was $68 \%$, the recall was $68 \%$ and F-measure was $67 \%$, the accuracy of the whole evaluation was $60 \%$. There were a total of 454 reviews. Positive reviews were 186 and 163 are identified correctly. Total negative were 164 and 153 are identified correctly and total non-related reviews were 104 and 103 are identified successfully. There were different causes for the deviation of results from the original results. One main reason is no predefined rules for posting reviews in roman Urdu. The user has not used proper rules and spelling for posting reviews. So some of the words in negative lexicon also match the words present in positive reviews. For example, the word "kam" is present in a negative lexicon also present in positive review "Ye mobile acha hai or price bhi bohat kam aur affordable he very nice" so because of this reason many positive reviews are identified 
TABLE 5.3

Contingency table results

\begin{tabular}{|c|c|c|c|c|c|}
\hline Products & Total & TP & FP & TN & FN \\
\hline Noise & 104 & 103 & 0 & 0 & 1 \\
\hline Samsung & 82 & 18 & 14 & 19 & 31 \\
\hline Qmobile & 170 & 45 & 64 & 29 & 32 \\
\hline Nokia & 96 & 33 & 15 & 23 & 27 \\
\hline Total & 452 & 199 & 93 & 71 & \\
\hline
\end{tabular}

TABLE 5.4

Final Results

\begin{tabular}{|c|c|c|c|c|}
\hline Total Reviews & Precision & Recall & F-measure & Accuracy \\
\hline 199 & 0.68 & 0.68 & .68 & 0.60 \\
\hline
\end{tabular}

TABLE 5.5

Boolean rules

\begin{tabular}{|c|c|c|c|c|}
\hline Rule no & \multicolumn{2}{|c|}{ Input } & Output & Output Gate \\
\hline 1 & True & False & False & AND \\
\hline 2 & True & True & True & EXNOR \\
\hline 3 & False & False & True & EXNOR \\
\hline 4 & False & True & False & AND \\
\hline 5 & \multicolumn{2}{|c|}{ Positive } & Positive & Single gate \\
\hline 6 & \multicolumn{2}{|c|}{ Negative } & Negative & Single gate \\
\hline
\end{tabular}

TABLE 5.6

No of words of all lexicon

\begin{tabular}{|c|c|}
\hline Lexicon & No of word \\
\hline Noise lexicon & 45 \\
\hline Positive lexicon & 50 \\
\hline Negative lexicon & 55 \\
\hline
\end{tabular}

TABLE 5.7

Shows the noise lexicon

\begin{tabular}{|c|l|}
\hline Noise lexicon words & \multicolumn{1}{|c|}{ Reviews } \\
\hline \multirow{3}{*}{ Warranty } & $\begin{array}{l}\text { mery pas lumia 530 hy warranty nhihy full box hy } \\
\text { kisi ko chahye tomsg kry no call only sms plzzfinal } \\
4200 \text { iam from multan 0301_****** }\end{array}$ \\
\hline \multirow{2}{*}{ Sale } & $\begin{array}{l}\text { Me j5 mobile sale krna chata ho. Condition 9/10 } \\
\text { white colorprice in16000 location Faisalabad sell } \\
\text { no 0321-******* }\end{array}$ \\
\hline
\end{tabular}

TABle 5.8

Positive lexicon

\begin{tabular}{|c|l|}
\hline Positive lexicon words & \multicolumn{1}{c|}{ Positive Reviews } \\
\hline Zabardast & $\begin{array}{l}\text { Zabardast mobile ha batterytiming ny to } \\
\text { tamam smart phones kopechy chor dia }\end{array}$ \\
\hline Outclass & $\begin{array}{l}\text { Outclass mobile he. aik din batrery backup. } \\
\text { camera front and back bhtacha } \mathrm{h}\end{array}$ \\
\hline
\end{tabular}

TABLE 5.9

Negative lexicon

\begin{tabular}{|c|c|}
\hline Negative lexicon words & Negative Reviews \\
\hline Bura & yar ya mobile kisi kam ka naihay bht hi bura hay \\
\hline Bekar & $\begin{array}{c}\text { full time bekar set ha yah 3.30ghanty laita ha } \\
\text { charging ma ... Paisybrbad krny ha tw ly lo }\end{array}$ \\
\hline
\end{tabular}


TABLE 5.10

Contingency matrix of Samsung mobile

\begin{tabular}{|c|c|c|c|}
\hline \multicolumn{2}{|c|}{} & \multicolumn{2}{c|}{ Prediction } \\
\cline { 3 - 4 } Actual & Negative & Negative & Positive \\
\cline { 2 - 4 } & Positive & 31 & 14 \\
\hline
\end{tabular}

TABLE 5.11

Total Result of Samsung mobile

\begin{tabular}{|c|c|c|c|c|}
\hline Total Reviews & Precision & Recall & F-measure & Accuracy \\
\hline 96 & 0.56 & 0.36 & 0.43 & 0.45 \\
\hline
\end{tabular}

TABLE 5.12

Contingency table of Nokia mobile

\begin{tabular}{|c|c|c|c|}
\hline \multicolumn{2}{|c|}{} & \multicolumn{2}{c|}{ Prediction } \\
\cline { 3 - 4 } \multicolumn{2}{|c|}{} & Negative & Positive \\
\hline \multirow{2}{*}{ Actual } & Negative & 23 & 15 \\
\cline { 2 - 4 } & Positive & 27 & 33 \\
\hline
\end{tabular}

TABLE 5.13

Total Result of Nokia mobile

\begin{tabular}{|c|c|c|c|c|}
\hline Total Reviews & Precision & Recall & F-measure & Accuracy \\
\hline 96 & 0.56 & 0.36 & 0.43 & 0.45 \\
\hline
\end{tabular}

TABLE 5.14

Contingency table of $Q$ mobile

\begin{tabular}{|c|c|c|c|}
\hline & \multicolumn{2}{|c|}{ Prediction } \\
\hline & & Negative & Positive \\
\hline \multirow{2}{*}{ Actual } & Negative & 29 & 64 \\
\hline & Positive & 32 & 45 \\
\hline
\end{tabular}

TABLE 5.15

Total Result of $Q$ mobile

\begin{tabular}{|c|c|c|c|c|}
\hline Total Reviews & Precision & Recall & F-measure & Accuracy \\
\hline 170 & 0.41 & 0.58 & 0.48 & 0.43 \\
\hline
\end{tabular}

TABLE 5.16

Total Result of all products

\begin{tabular}{|c|c|c|c|c|}
\hline Total Reviews & Precision & Recall & F-measure & Accuracy \\
\hline 170 & 0.50 & 0.56 & 0.51 & 0.47 \\
\hline
\end{tabular}

TABLE 5.17

Contingency table results of all products

\begin{tabular}{|c|c|}
\hline Orientation & Number of comments \\
\hline True Positive (TP) & 96 \\
\hline False positive (FN) & 93 \\
\hline True Negative (TN) & 70 \\
\hline False Negative (FN) & \\
\hline
\end{tabular}

TABLE 5.18

Final results of all products

\begin{tabular}{|c|c|c|c|c|c|c|}
\hline Serial no & Products & Total & TP & FP & TN & FN \\
\hline 1 & Samsung & 81 & 18 & 14 & 19 & 31 \\
\hline 2 & Qmobile & 170 & 45 & 64 & 29 & 32 \\
\hline 3 & Nokia & 96 & 33 & 15 & 23 & 27 \\
\hline 4 & Total & 348 & 96 & 93 & 71 & 90 \\
\hline
\end{tabular}




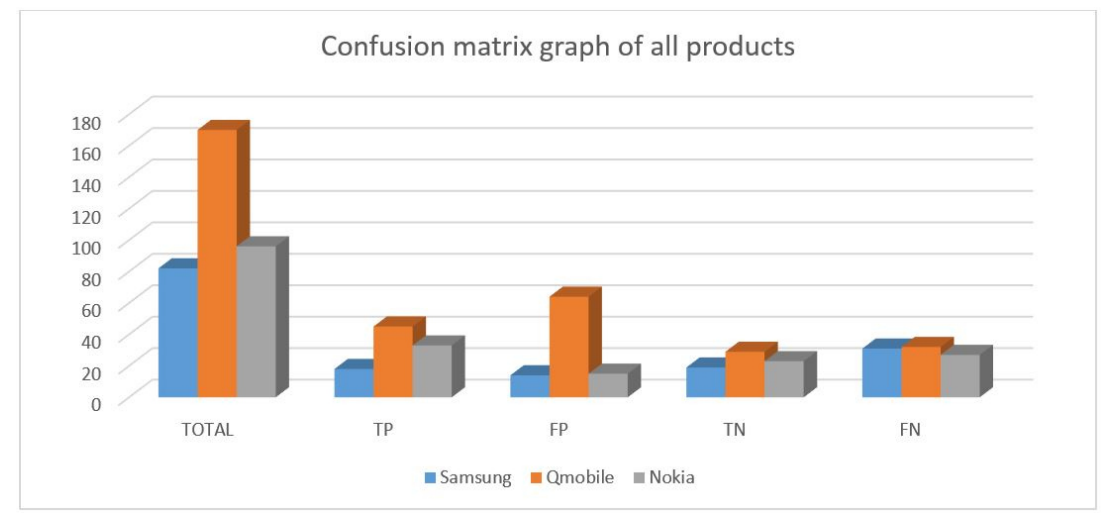

FIG. 5.5. Contingency matrix graph of $n$ Reviews

as negative. The second main reason for result deviation was Boolean rules that were created for identification of review that consist of double negative words i.e. rules no 3 . Rule no 3 was made for identification of reviews that consist of two negative words for example "bura" and "nahi". And the reviews were identified as positive, but there were some other reviews which are consist of some more negative words "3rdclass" and "bekar". So, these two words were also present in different reviews and that are identified as positive because of rule no 3 .

7.1. Recommendations. The model gave satisfactory results up to some extent. Precision was $50 \%$ and more enhancement could be achieved by implementing new methods for handling different errors. Errors and inconsistencies can be handled in different ways. Improving Boolean rules can increase the model accuracy. All the lexicons are designed manually which did not cover the whole lexicons and could not achieve the perfect result, instead of manually if semantic lexicon will use such as wordnet it will give a much better result.

7.2. Future work. In the future we saggested the accuracy of the model can be increased by using a wordnet lexicon generator. Secondly, the accuracy can be increased by identifying the word that changes the polarity of the words from negative to positive and vice versa. From the research, it is found that the word "nahi" changed the polarity from negative to positive and vice versa. Improvement can be done by improving the word "nahi" and making a separate code for this word to evaluate it much better.

Authors' contributions:. All the authors contributed to this research. The order of authors in this manuscript is maintained depending on the level of contributions they made in this research.

\section{REFERENCES}

[1] Hall, Mark and Frank, Eibe and Holmes, Geoffrey and Pfahringer, Bernhard and Reutemann, Peter and Witten, IAN H, The WEKA data mining software: an update, ACM SIGKDD explorations newsletter, 11, 1, (2009), pp. 10-18.

[2] Dave, Kushal and Lawrence, Steve and Pennock, David M, Mining the peanut gallery: Opinion extraction and semantic classification of product reviews, Proceedings of the 12th international conference on World Wide Web, (2003), pp. 519528.

[3] Hu, Minqing And Liu, Bing,Mining and summarizing customer reviews, Proceedings of the tenth ACM SIGKDD international conference on Knowledge discovery and data mining, pp. 168-177, 2004.

[4] Daud, Misbah and Khan, Rafiullah and Daud, Aitazaz and others, Roman Urdu opinion mining system (RUOMiS), arXiv preprint arXiv:1501.01386, 2015

[5] Znhang, Kunpeng and Narayanan, Ramanathan and Choudhary, Alok N, Voice of the Customers: Mining Online Customer Reviews for Product Feature-based Ranking., WOSN, 10, pp. 11-11, 2010

[6] Abbasi, Ahmed and Chen, Hsinchun and Salem, Arab Sentiment analysis in multiple languages: Feature selection for opinion classification in web forums, ACM Transactions on Information Systems (TOIS), 26, 3, pp. 1-34, 2008,ACM New York, NY, USA

[7] Han, Jiawei and Pei, Jian and Kamber, Micheline, Data mining: concepts and techniques, 2011, Elsevier

[8] Wilson, Theresa and Wiebe, Janyce and Hoffmann, Paul, Recognizing contextual polarity in phrase-level sentiment analysis, Proceedings of human language technology conference and conference on empirical methods in natural language processing, pp. 347-354, 2005 
[9] Cercel, Dumitru-Clementin and Trăuşan, Ştefan, Research Challenges in Opinion Mining From A Natural Language Processing Perspective, University Politehnica of Bucharest Scientific Bulletin Series C-Electrical Engineering aAnd Computer Science, 78, 3, pp. 157-168, 2016

[10] Rashid, Ayesha and Anwer, Naveed and Iqbal, Muddaser and Sher, Muhammad, A survey paper: areas, techniques and challenges of opinion mining, International Journal of Computer Science Issues (IJCSI), 10, 6, pp. 18, 2013, Citeseer

[11] Kouloumpis, Efthymios and Wilson, Theresa And Moore, Johanna, Twitter sentiment analysis: The good the bad and the omg!, Fifth International AAAI conference on weblogs and social media, 2011

[12] Mahar, Javed Ahmed and Memon, Ghulam Qadir, Rule based part of speech tagging of sindhi language, 2010 International Conference on Signal Acquisition and Processing, pp. 101-106, 2010, IEEE

[13] Pak, Alexander and Paroubek, Patrick, Twitter as a corpus for sentiment analysis and opinion mining., LREc, 10, 2010, pp. 1320-1326, 2010

[14] Wang, Dingding And Zhu, Shenghuo and Li, Tao, SumView: A Web-based engine for summarizing product reviews and customer opinions, Expert Systems with Applications, 40, 1, pp. 27-33, 2013, Elsevier

[15] Yassine, Mohamed and HajJ, Hazem, A framework for emotion mining from text in online social networks, 2010 IEEE International Conference on Data Mining Workshops, pp. 1136-1142, 2010, IEEE

[16] Ha jmohammadi, Mohammad Sadegh and Ibrahim, Roliana and Othman, Zulaiha Ali, Opinion mining and sentiment analysis: A survey, International Journal of Computers \& Technology, 2, 3, pp. 171-178, 2012

[17] von Helversen, Bettina and Abramczuk, Katarzyna and Kopeć, Wieseaw and Nielek, Radoslaw, Influence of consumer reviews on online purchasing decisions in older and younger adults, Decision Support Systems, 113, pp. 1-10, 2018, Elsevier

[18] Du, Rundong and Lu, Zhongming and Pandit, Arka and Kuang, Da and Crittenden, John and Park, Haesun, Toward Social Media Opinion Mining for Sustainability Research, Workshops at the Twenty-Ninth AAAI Conference on Artificial Intelligence, 2015

[19] LiU, BING, Sentiment analysis and opinion mining, Synthesis lectures on human language technologies, 5, 1, pp. 1-167, 2012, Morgan \& Claypool Publishers

[20] Agarwal, Apoorv and Xie, Boyi and Vovsha, Ilia and Rambow, Owen and Passonneau, Rebecca J, Sentiment analysis of twitter data, Proceedings of the workshop on language in social media (LSM 2011), pp. 30-38, 2011

[21] El-Halees, AlaA M, Arabic opinion mining using combined classification approach, Arabic opinion mining using combined classification approach, 2011, Naif Arab University for Security Sciences

[22] N-gram based approach for opinion mining of Punjabi text, KaUR, AmandeEP AND Gupta, Vishal, International Workshop on Multi-disciplinary Trends in Artificial Intelligence, pp. 81-88, 2014, Springer

[23] Bilal, Muhammad and Israr, Huma and Shahid, Muhammad and Khan, Amin, Sentiment classification of Roman-Urdu opinions using Nä̈ve Bayesian, Decision Tree and KNN classification techniques, Journal of King Saud UniversityComputer and Information Sciences, 28, 3, pp. 330-344, 2016, Elsevier

[24] Li, NAn AND Wu, Desheng Dash, Using text mining and sentiment analysis for online forums hotspot detection and forecast, Decision support systems, 48, 2, pp. 354-368, 2010, Elsevier

Edited by: P. Vijaya

Received: Nov 26, 2019

Accepted: Jun 26, 2020 